\title{
[14] In Vitro Reconstitution of Cdc42-Mediated Actin Assembly Using Purified Components
}

\author{
By Hsin-Yi Henry Ho, Rajat Rohatgi, \\ Andres M. Lebensohn, and Marc W. Kirschner
}

\begin{abstract}
In the accompanying chapter, we describe an in vitro system that uses Xenopus egg extracts to study actin assembly induced by phosphatidylinositol $(4,5)$ bisphosphate $\left(\mathrm{PIP}_{2}\right)$ and $\mathrm{Cdc} 42$. Biochemical fractionation and candidate screening experiments conducted in the extract system have identified the Arp2/3 complex, the N-WASP-WIP (or N-WASP-CR16) complex, and the Cdc42-binding protein Toca- 1 as important mediators of $\mathrm{PIP}_{2}$ - and $\mathrm{Cdc} 42$-actin signaling. Toward our ultimate goal of reconstituting an in vitro system that recapitulates the signaling properties observed in vivo, we then developed a purified actin assembly assay system consisting of the regulatory components that we discovered from extracts. In these assays, the stereotypical sigmoidal kinetics of actin polymerization are monitored by pyrene-actin fluorescence in the presence of defined recombinant or purified proteins, enabling the detailed study of mechanism and protein function. In this chapter, we describe the preparation of the components used in these purified actin assembly reactions, as well as the assay conditions under which we monitor actin polymerization kinetics in vitro.
\end{abstract}

\section{Introduction}

In the previous chapter, we described an in vitro system that uses total cytoplasmic extracts made from Xenopus eggs to monitor actin assembly induced by phosphatidylinositol $(4,5)$ bisphosphate $\left(\mathrm{PIP}_{2}\right)$ and Cdc42. The extract systems are very useful for determining requirements for particular components, using either biochemical fractionation or immunodepletion/ add-back of candidates. However, to establish sufficiency and to explain the direct interactions between components, we have developed purified actin assembly systems based on the well-established pyrene-actin assay (Cooper et al., 1983; Kouyama and Mihashi, 1980). In these assays, the stereotypical sigmoidal kinetics of pyrene-labeled pure actin is followed in the presence of defined (either recombinant or purified) proteins. Our overall strategy in studying this signaling pathway has been to first identify required proteins using the extract system and then to move to the purified 
system to determine the mechanism and protein function, with the ultimate goal of building a reconstituted system that reflects (as closely as possible) the signaling properties in vivo.

We describe here two purified signaling modules that link Cdc42 and $\mathrm{PIP}_{2}$ to the actin nucleating Arp2/3 complex. In the first system (called Reaction \#1 henceforth), Cdc42 and PIP $_{2}$ synergistically activate N-WASP, which in turn stimulates the actin nucleating activity of the Arp2/3 complex. This is a remarkably parsimonious module that shows how the activation properties of N-WASP can dictate actin polymerization precisely at the temporal and spatial intersection of two signals. Since its original report in 1999 (Rohatgi et al., 1999), Reaction \#1 has been widely used to study mechanistic aspects of $\mathrm{N}-\mathrm{W} A S P$ regulation, including the autoinhibition of N-WASP and the cooperative mechanism of N-WASP activation by $\mathrm{Cdc} 42$ and $\mathrm{PIP}_{2}$ (Higgs and Pollard, 2000; Rohatgi et al., 2000). In addition, it has served as an assay to identify additional regulators of N-WASP activity, such as the adaptor proteins Nck/Grb2 and tyrosine kinases (Carlier et al., 2000; Cory et al., 2002; Rohatgi et al., 2001; Suetsugu et al., 2002; Torres and Rosen, 2003).

Despite the insights gained from studying Reaction \#1, detailed analyses of the extracts system have suggested that the Cdc42 signaling pathway is significantly more complicated and involves additional levels of regulation. To understand the molecular logic of this complexity, we have recently identified (through biochemical purification from extracts) two additional components of the $\mathrm{Cdc} 42$ pathway, an effort that has allowed us to describe a more complex purified assay system (Reaction \#2) (Ho et al., 2004). These components include the protein WIP or the closely related CR16, which exists in a tight complex with N-WASP in these extracts and the novel Cdc42 binding protein Toca-1 (Ho et al., 2001, 2004; Ramesh et al., 1997). We believe that Reaction \#2 represents a more physiological recapitulation of the Cdc42 pathway in vivo, because it exhibits a number of features that are characteristic of the complete extract system but are lacking in our original Reaction \#1.

In this chapter, we describe the preparation of the components used in Reactions \#1 and \#2, as well as the assay system used to monitor in vitro the actin assembly kinetics in these reactions. Finally, some properties of these reactions are discussed.

\section{Preparation of Reaction Components}

\section{Preparation of Recombinant $N$-WASP}

We have used untagged recombinant full-length N-WASP (rat origin) in our purified actin polymerization system (Reaction \#1). Because a major use of these assays is to study N-WASP activation, it is critical to use 
protein preparations that show low levels of basal activity toward the Arp2/ 3 complex. Several affinity-tagged versions of N-WASP have been made in our laboratory, but the activities of these fusion proteins are quite variable depending on the epitope used. Myc(x6)-N-WASP and His(x6)-N-WASP exhibit significant levels of basal activation even in the absence of activators and are, therefore, less useful for most applications (unpublished observations). GST-N-WASP behaves more similarly to untagged $\mathrm{N}$-WASP in that it has a relatively low basal activity that can be further stimulated by $\mathrm{Cdc} 42$ and $\mathrm{PIP}_{2}$; however, this protein has the caveat that it is dimerized through the GST moiety (untagged N-WASP is a monomer [Rohatgi et al., 2000]). For these reasons, we have used the untagged protein in most of our experiments that involve recombinant N-WASP.

Untagged N-WASP is expressed in Spodoptera frugiperda (Sf9) cells using the Bac-to-Bac baculoviral protein expression system (Invitrogen) and purified using a three-step conventional purification scheme modified from Miki et al. (1996) and Rohatgi et al. (1999) (Fig. 1). We have not been able to produce recombinant full-length N-WASP in Escherichia coli. All chromatographic steps are performed using an FPLC liquid handling system (Amersham Biosciences) equipped with columns purchased from the same supplier. The following procedure is based on a typical preparation that begins with 31 of Sf 9 culture, yielding approximately $10 \mathrm{mg}$ of highly purified N-WASP. With proper preparation, the entire purification can be completed in 1 long day.

Sf9 cells are infected with a recombinant baculovirus overexpressing the N-WASP protein at a density of 1 million cells $/ \mathrm{ml}$. Cells are harvested $72 \mathrm{~h}$ after infection by centrifugation, washed once with PBS ( $20 \mathrm{mM}$ $\mathrm{Na}_{2} \mathrm{HPO}_{4}, 2.8 \mathrm{~m} M \mathrm{KH}_{2} \mathrm{PO}_{4}$, pH 7.3, $140 \mathrm{mM} \mathrm{NaCl}, 2.7 \mathrm{mM} \mathrm{KCl}$ ) and resuspended in $200 \mathrm{ml}$ lysis buffer $(20 \mathrm{~m} M$ Na-Tris, pH 7.5, $20 \mathrm{mM} \mathrm{NaCl}$, $2 \mathrm{~m} M$ EDTA, $1 \mathrm{~m} M$ PMSF, $1 \mathrm{~m} M$ DTT, $10 \mu \mathrm{g} / \mathrm{ml}$ each leupeptin/pepstatin/ chymostatin, $1 \times$ complete protease inhibitor cocktail [tablets with EDTA, Roche]). Cells are lysed by sonication, and the lysate is cleared by centrifugation at $\sim 160,000 \mathrm{~g}(45,000 \mathrm{rpm}$ in a Beckman Type 45 Ti rotor) for $1 \mathrm{~h}$. The supernatant is filtered through a $0.45-\mu \mathrm{m}$ syringe filter (Millipore), loaded on two 5-ml HiTrap Heparin columns connected in series, and $\mathrm{N}$-WASP is eluted with a linear gradient of $0-700 \mathrm{mM} \mathrm{NaCl}$ developed over 10 column volumes. N-WASP elutes at approximately $600 \mathrm{mM}$ $\mathrm{NaCl}$ and can be identified on a Gelcode Blue (Pierce)-stained gel as a prominent protein band migrating at $\sim 66 \mathrm{kDa}$ (see Fig. 1).

The peak N-WASP-containing fractions are pooled and dialyzed against 21 of $20 \mathrm{~m} M$ Na-Tris, $\mathrm{pH} 8.0,50 \mathrm{~m} M \mathrm{NaCl}, 1 \mathrm{~m} M$ DTT for $1 \mathrm{~h}$ (some precipitation may occur). After the dialysis, the protein sample is diluted with an equal volume of buffer Q-A (20 mM Na-Tris, pH 8.3, 
$50 \mathrm{~m} M \mathrm{NaCl}, 1 \mathrm{~m} M$ DTT) and centrifuged at $\sim 180,000 \mathrm{~g}(50,000 \mathrm{rpm}$ in a Beckman Type $70 \mathrm{Ti}$ rotor) for $15 \mathrm{~min}$. The supernatant is filtered through a $0.45-\mu \mathrm{m}$ syringe filter (Millipore) and loaded onto a 5-ml HiTrap Q column, and N-WASP is eluted with a linear gradient of $50-700 \mathrm{mM} \mathrm{NaCl}$ over 10 column volumes. N-WASP elutes at $200-250 \mathrm{mM} \mathrm{NaCl}$, and can be identified on a Gelcode Blue-stained gel as the predominant protein (Fig. 1).

The peak N-WASP-containing fractions are pooled and loaded onto a 120-ml HiLoad Superdex 200 16/60 gel filtration column pre-equilibrated with gel filtration buffer (20 m $M$ HEPES, pH 7.7, $100 \mathrm{~m} M \mathrm{KCl}, 1 \mathrm{~m} M$ DTT). The column is run at a flow rate of $0.5 \mathrm{ml} / \mathrm{min}$, and N-WASP elutes as a single symmetric peak at approximately $64 \mathrm{ml}$. The peak N-WASP fraction has a concentration of $\sim 1 \mathrm{mg} / \mathrm{ml}$ determined by the Bradford assay (BioRad), with a purity of $>95 \%$ as judged by SDS PAGE and Gelcode Blue staining (Fig. 1). Fractions with protein concentrations lower than $1 \mathrm{mg} / \mathrm{ml}$ are supplemented with glycerol to a final concentration of $10 \%$ $(\mathrm{v} / \mathrm{v})$. N-WASP fractions are divided into single-use aliquots, snap frozen in liquid nitrogen, and stored at $-80^{\circ}$.

\section{Purification of Native N-WASP-WIP Complex from Xenopus Egg Extracts}

Most of the N-WASP in extracts is present as a stable complex with WIP or CR16 (depending on the tissue source), and analysis of the Cdc42Arp2/3-actin pathway required production of purified complex (Ho et al., 2001, 2004; Ramesh et al., 1997 ). Despite efforts using insect cells and cultured mammalian cells, we have not been able to produce a complex that exhibits proper regulation using recombinant techniques. Thus, native N-WASP-WIP complex is purified conventionally from the high-speed supernatant (HSS) made from Xenopus eggs (Fig. 2). We have previously described the isolation of native N-WASP-CR16 complex from bovine brain extracts (Ho et al., 2001), but it requires a more difficult purification scheme because of the tissue heterogeneity of this starting material.

Throughout the purification, the N-WASP-WIP complex is followed by Western blotting with a rabbit polyclonal $\alpha$-N-WASP antibody (Rohatgi et al., 1999). All chromatographic media are purchased from Amersham Biosciences. Xenopus egg HSS is prepared from $150 \mathrm{frogs}$ as described in the previous chapter, except that the crude cytoplasmic extract $(\sim 250 \mathrm{ml})$ is diluted only fivefold before high-speed ultracentrifugation and is not reconcentrated. Given the poor recovery of the complex, this purification scale is necessary to produce sufficient final protein yield $(\sim 0.5 \mathrm{mg})$ and purity (>95\%). The HSS is first fractionated by performing a $20-35 \%$ 
A

Lysate from Sf9 cells overexpressing untagged N-WASP

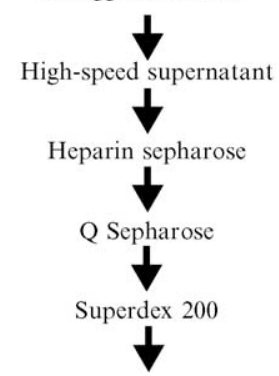

Purified N-WASP

C

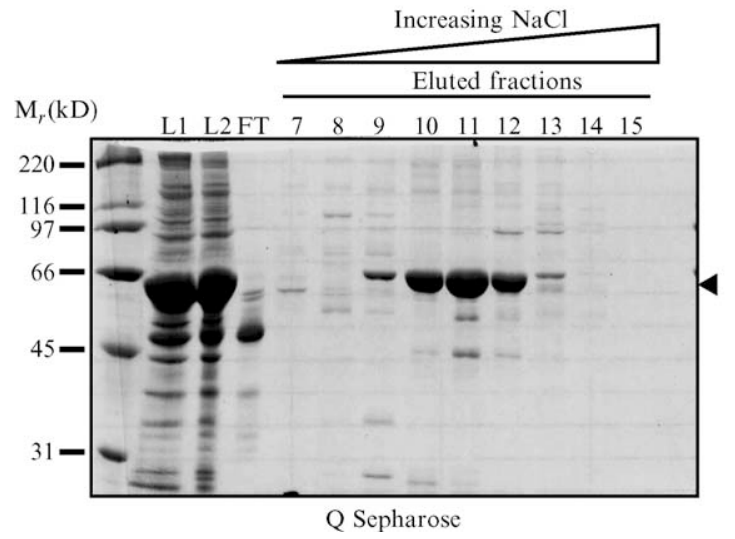

B

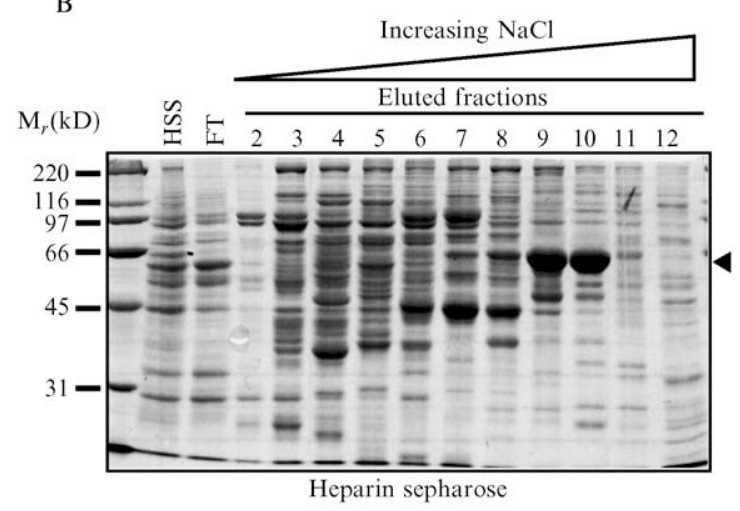

D

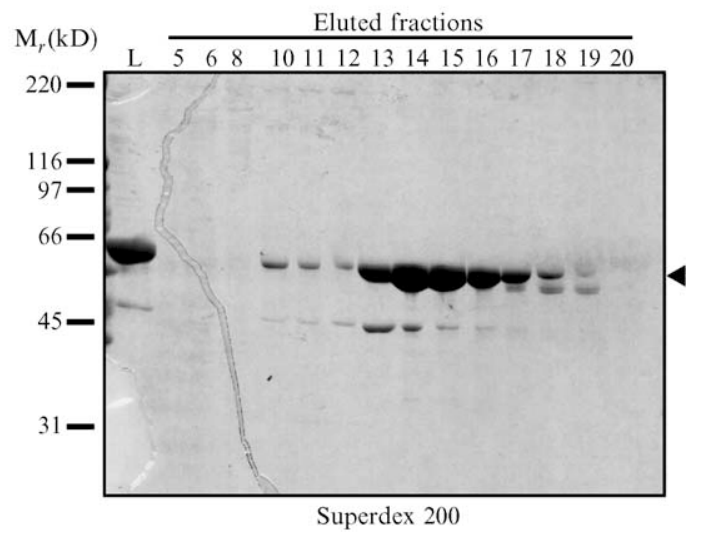

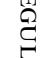

$\sum_{3}$

윰

젹

菊

寜

워

?

E

$\Omega$

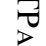

量

n.

짐

궁

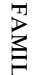

$\Xi$ 
ammonium sulfate cut. The precipitate is collected by centrifugation $(21,000 \mathrm{~g}$ or $15,000 \mathrm{rpm}$ in a Beckman Type $19 \mathrm{Ti}$ rotor for $1 \mathrm{~h})$, resuspended in $140 \mathrm{ml}$ of buffer CSF-XB $(100 \mathrm{mM} \mathrm{KCl}, 2 \mathrm{mM} \mathrm{MgCl} 2,10 \mathrm{~m} M \mathrm{~K}-$ HEPES, $\mathrm{pH} 7.7,50 \mathrm{~m} M$ sucrose, $5 \mathrm{~m} M$ EGTA), and dialyzed into buffer QA (30 $\mathrm{m} M$ Na-Tris, pH 7.8, $100 \mathrm{~m} M \mathrm{KCl}, 1 \mathrm{~m} M$ EDTA, $1 \mathrm{~m} M$ DTT). The dialyzed protein sample is centrifuged at $265,000 \mathrm{~g}(60,000 \mathrm{rpm}$ in a Beckman Type 70 Ti rotor) for $1 \mathrm{~h}$ at $4^{\circ}$, and the supernatant is filtered through $0.22-\mu \mathrm{m}$ syringe filters (Millipore). The fraction is then loaded onto a $66-\mathrm{ml}$ Q Sepharose HP column (poured in a XK 26/40 column) and eluted with a linear gradient of $100-400 \mathrm{mM} \mathrm{KCl}$ developed over 10 column volumes. Peak N-WASP-containing fractions (eluting at $\sim 200 \mathrm{mM} \mathrm{NaCl}$ ) are pooled and dialyzed against buffer HA (20 mM Na-Tris, pH 7.5, $100 \mathrm{~m} M$ $\mathrm{NaCl}, 1 \mathrm{~m} M$ EDTA, $1 \mathrm{~m} M$ DTT) and loaded onto two 5-ml HiTrap Heparin HP columns connected in series. N-WASP-WIP is eluted from the heparin column with a linear gradient of 100-700 $\mathrm{mM} \mathrm{NaCl}$ developed over 10 column volumes. Peak N-WASP-containing fractions (eluting at $\sim 400 \mathrm{~m} M \mathrm{NaCl})$ are pooled and dialyzed into buffer SA $(20 \mathrm{~m} M$ Na-PIPES, pH 6.8, $80 \mathrm{~m} M \mathrm{NaCl}, 10 \%$ glycerol, $5 \mathrm{~m} M \beta$-octylglucoside, 1 $\mathrm{m} M$ DTT) and centrifuged at $16,700 \mathrm{~g}(20,000 \mathrm{rpm}$ in a Beckman TLA 100.4 rotor) for $10 \mathrm{~min}$ to remove particulates. The material is loaded onto a 1-ml Mono S column, eluted with a linear gradient of $80-400 \mathrm{mM} \mathrm{NaCl}$ developed over 20 column volumes (N-WASP elutes at $\sim 200 \mathrm{mM} \mathrm{NaCl}$, see Fig. 2), and the fractions are mixed immediately with the appropriate volume of $1.5 \mathrm{M}$ Tris base to bring to a final $\mathrm{pH}$ of 7.2. Before proceeding to the final sucrose gradient step, the peak N-WASP-containing fractions are pooled and concentrated so that all of the material can be loaded on a single $12-\mathrm{ml}$ sucrose gradient. This is performed by binding the protein to a 0.1-ml Mono S column (Amersham Biosciences, Mono S PC 1.6/5 for SMART system; the sample is first diluted fourfold in buffer SA before applying to the column) and eluting it with a step gradient of $400 \mathrm{mM}$ $\mathrm{NaCl}$. The concentrated material is then loaded onto a $12-\mathrm{ml} 5-20 \%(\mathrm{w} / \mathrm{v})$ sucrose gradient poured in buffer XB (30 $\mathrm{m} M$ K-HEPES, pH 7.7, $100 \mathrm{~m} M$ $\mathrm{KCl}, 1 \mathrm{~m} M \mathrm{MgCl}_{2}, 0.1 \mathrm{~m} M$ EDTA) plus $5 \mathrm{~m} M \beta$-octylglucoside and $1 \mathrm{~m} M$ DTT. The sucrose gradient is centrifuged at $40,000 \mathrm{rpm}$ for $17 \mathrm{~h}$ in a Beckman SW40 swinging-bucket rotor. The two subunits of the complex,

FIG. 1. Purification of recombinant N-WASP from Sf9 cells. (A) The fractionation scheme used to purify untagged N-WASP expressed recombinantly in Sf9 cells. (B-D) The protein composition of fractions from the various stages of the purification analyzed by SDS-PAGE and Gelcode Blue staining. The position of N-WASP is indicated by the arrowhead on the right side of each gel. (B) HSS, high-speed supernatant; FT, flow-through. (C) L1, load material before dialysis; L2, load material after dialysis; FT, flow-through. (D) L, load. 
A

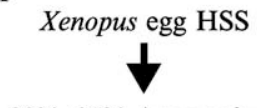

20\%-35\% Ammonium sulfate cut

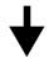

Q Sepharose

$\downarrow$

Heparin-sepharose

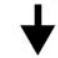

Mono S

I

$5 \%-20 \%$ Sucrose

gradient

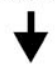

N-WASP-WIP complex
B
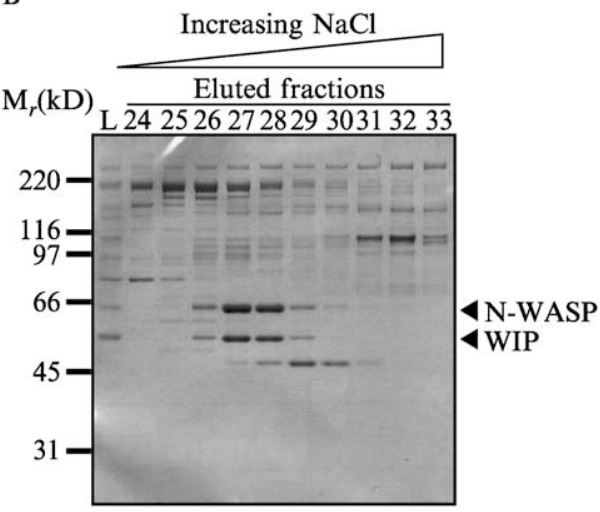

Mono S

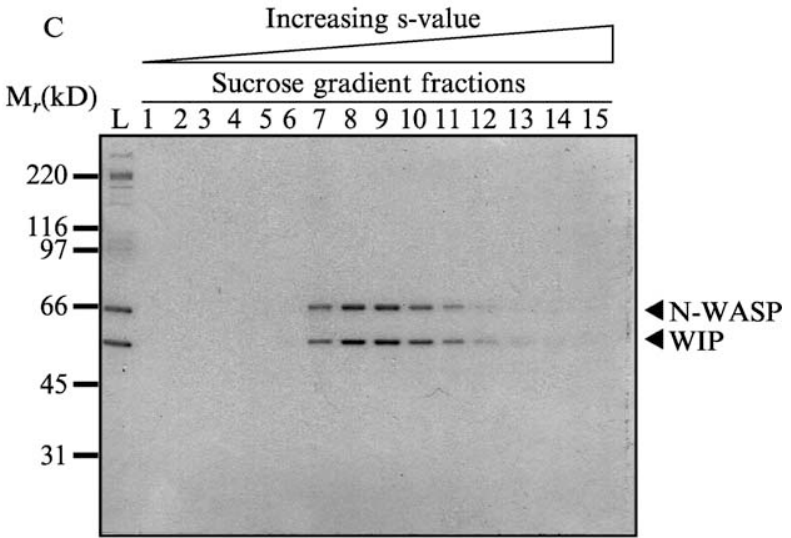

Sucrose gradient

FIG. 2. Purification of native N-WASP-WIP complex from Xenopus egg extracts. (A) The fractionation scheme used to purify N-WASP-WIP complex from Xenopus egg extracts. (B) The protein composition of fractions from the Mono $S$ step of the purification analyzed by SDS-PAGE and Gelcode Blue staining. The positions of N-WASP and WIP are indicated by the arrowhead on the right. (C) Fractions from the final sucrose gradient step of the purification analyzed by SDS-PAGE and Gelcode Blue staining. Again, the positions of $\mathrm{N}-\mathrm{WASP}$ and WIP are indicated by the arrowhead on the right. L, load.

N-WASP and WIP, are identified on a Gelcode Blue-stained SDS polyacrylamide gel as the only detectable protein bands (Fig. 2). Purified $\mathrm{N}$-WASP-WIP complex is stored on ice and used in actin polymerization 
assays within $48 \mathrm{~h}$. The sucrose gradient fractions can also be divided into single-use aliquots, snap frozen in liquid nitrogen, and stored at $-80^{\circ}$. We have found that one freeze-thaw cycle does not significantly affect the activity of the complex in actin polymerization assays.

\section{Preparation of Recombinant Toca-1}

The wild-type cDNA encoding human Toca-1 is lethal to E. coli. Although the nature of this toxicity has remained unknown, we have generated a nontoxic form of human Toca-1 DNA (H-form) by introducing silent wobble-position mutations to codons 126 and 127 (Ho et al., 2004) (Fig. 3). The $\mathrm{H}$-form of human Toca-1 is tagged on its $\mathrm{N}$-terminus with a hexahistadine tag and expressed in insect (Sf9) cells using the Bac-to-Bac baculoviral protein expression system (Invitrogen) and purified in one step over a nickel column. Despite considerable efforts, we have not been able to express recombinant human Toca-1 in E. coli.

Three hundred milliliters of Sf9 cells at a density of 1 million cells $/ \mathrm{ml}$ are infected with the baculovirus overexpressing Toca-1. Cells are harvested $72 \mathrm{~h}$ after infection by centrifugation and washed once in PBS. The cell pellet is resuspended in $40 \mathrm{ml}$ lysis buffer $\left(50 \mathrm{mM} \mathrm{NaH} \mathrm{PO}_{4}, \mathrm{pH}\right.$ 8.0, $300 \mathrm{~m} M \mathrm{NaCl}, 10 \mathrm{~m} M$ imidazole, $1 \%$ Triton X-100, $5 \mathrm{~m} M \beta$-mercaptoethanol, $1 \mathrm{~m} M$ PMSF, $1 \times$ protease inhibitor cocktail [tablets without EDTA, Roche]), homogenized 10 times using a glass/glass homogenizer with a small clearance pestle (Kontes Dounce Tissue Grinder, $40 \mathrm{ml}$, pestle B), and centrifuged at $\sim 100,000 \mathrm{~g}(36,000 \mathrm{rpm}$ in a Beckman Type $45 \mathrm{Ti}$ rotor) for $1 \mathrm{~h}$. The supernatant is collected and incubated in batch for $2 \mathrm{~h}$ to overnight at $4^{\circ}$ with $0.5 \mathrm{ml}$ of nickel agarose (Qiagen) pre-equilibrated with the lysis buffer. The beads are collected by passing the binding mixture through a 10-ml disposable column (BioRad) by gravity. The beads are washed in the column with 20-ml wash A $\left(50 \mathrm{mM} \mathrm{NaH}_{2} \mathrm{PO}_{4}, \mathrm{pH} 8.0,500\right.$ $\mathrm{m} M \mathrm{NaCl}, 5 \mathrm{~m} M$ imidazole, $0.5 \%$ Triton X-100, $5 \mathrm{~m} M \beta$-mercaptoethanol), followed by $20 \mathrm{ml}$ of wash $\mathrm{B}\left(50 \mathrm{~m} M \mathrm{NaH}_{2} \mathrm{PO}_{4}, \mathrm{pH} 8.0,300 \mathrm{~m} M \mathrm{NaCl}, 5 \mathrm{~m} M\right.$ imidazole, $0.5 \%$ Triton $\mathrm{X}-100,5 \mathrm{~m} M \beta$-mercaptoethanol). Toca- 1 is then eluted with 3-5 ml of elution buffer (50 $\mathrm{m} M \mathrm{NaH}_{2} \mathrm{PO}_{4}, \mathrm{pH} 8.0,300 \mathrm{~m} M$ $\mathrm{NaCl}, 255 \mathrm{~m} M$ imidazole, $5 \mathrm{~m} M \beta$-mercaptoethanol) in batch. Our typical yield is $3-5 \mathrm{mg}$ of protein from $300 \mathrm{ml}$ of Sf9 culture with a purity of $>95 \%$ as judged by Gelcode Blue staining (Fig. 3). Fractions with protein concentrations lower than $1 \mathrm{mg} / \mathrm{ml}$ are supplemented with glycerol to a final concentration of $10 \%(\mathrm{v} / \mathrm{v})$. Purified Toca-1 is aliquoted, snap frozen in liquid nitrogen, and stored at $-80^{\circ}$.

During preparative purifications of recombinant Toca-1, it is important to have at least $300 \mathrm{mM} \mathrm{NaCl}$ or $\mathrm{KCl}$ in all of the buffers, because Toca-1 
A

$$
\begin{aligned}
& \text { Nucleotides } 378 \quad 381 \\
& \text { H.s. Toca-1 (WT) --- } \frac{\text { caa }}{\mathrm{Q}} \frac{\text { tat }}{\mathrm{Y}} \frac{\mathrm{ctt}}{\mathrm{L}} \frac{\mathrm{gac}}{\mathrm{D}} \text {--- (toxic) } \\
& \text { H.s. Toca-1 (H-form) --- } \frac{\text { caa }}{\mathrm{Q}} \frac{\mathrm{tat}}{\mathrm{Y}} \frac{\mathrm{ctg}}{\mathrm{L}} \frac{\mathrm{gat}}{\mathrm{D}} \text {--- (non-toxic) } \\
& \text { Codons } 126127
\end{aligned}
$$

B

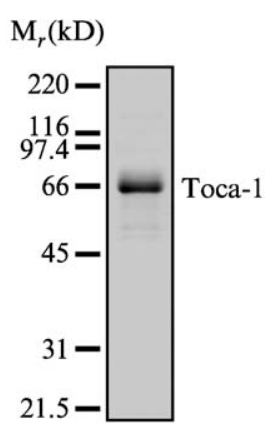

FIG. 3. Preparation of recombinant Toca-1. (A) The wild-type cDNA encoding Toca-1 is lethal to E. coli. Although the nature of this toxicity has remained unclear, we have generated a nontoxic form of human Toca-1 DNA (H-form) by introducing silent wobble-position mutations to codons 126 and 127 . The $\mathrm{H}$-form of human Toca- 1 is tagged on its $\mathrm{N}$-terminus with a hexahistadine tag, expressed in Sf9 cells and purified in one step over a nickel column. (B) A purified preparation of recombinant Toca-1 analyzed by SDS-PAGE and Gelcode Blue staining. (Reprinted with permission from Ho et al., 2004.)

(at a protein concentration between $0.1-1 \mathrm{mg} / \mathrm{ml}$ ) has a very low solubility under physiological salt concentrations. At a protein concentration of $>1 \mathrm{mg} / \mathrm{ml}$, even higher salt is needed to maintain the solubility of Toca- 1 . For actin polymerization reactions, we dilute Toca- 1 directly from stock solutions in $300 \mathrm{mM} \mathrm{NaCl}$ into the assay mixtures (which typically contain $100 \mathrm{~m} M \mathrm{KCl})$. Given that the final Toca-1 concentration in these reactions is quite low $(0.001-0.01 \mathrm{mg} / \mathrm{ml})$, it seems to remain in solution under these conditions.

\section{Purification of the Arp2/3 Complex from Bovine Brain Extracts}

Several different purifications of the Arp2/3 complex have been described in the literature (Machesky et al., 1994; Ma et al., 1998; Welch and Mitchison, 1998). For all of the assays described in this chapter, we have used Arp2/3 complex purified from bovine brain extracts by conventional column chromatography (Fig. 4). Perhaps the simplest way to purify the Arp2/3 complex has been published by Egile et al. (1999), who isolated the complex from bovine brain based on its affinity for the C-terminus of N-WASP. However, we have found that such preparations have higher basal level of activity in the purified assay system and are less specific than conventional purified complex.

The following procedure is based on a preparation that begins with 20 calf bovine brains, yielding approximately $10 \mathrm{mg}$ of purified Arp $2 / 3$ 
A

Bovine brai

Butyl Sepharose

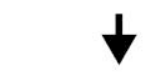

DEAE Sepharose

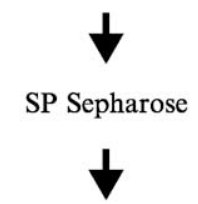

Resource phenyl

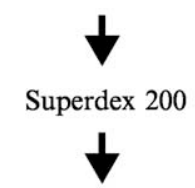

Arp2/3 complex
B

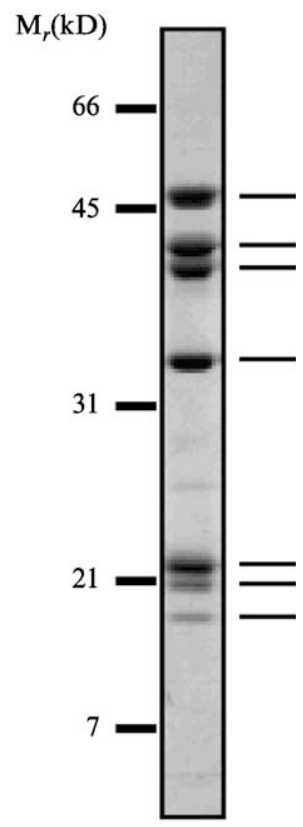

FIg. 4. Purification of the Arp $2 / 3$ complex from bovine brain extracts. (A) The fractionation scheme used to purify the Arp $2 / 3$ complex from bovine brain extracts. (B) The composition of the purified Arp2/3 complex shown on a $12 \%$ SDS polyacrylamide gel stained with Gelcode Blue. The thin, unlabeled lines on the right indicate the seven subunits of the complex. Listed in descending order of molecular weight, the subunits are Arp3, Arp2, p41-ARC, p34-ARC, p21-ARC, p20-ARC, and p16-ARC. (Reprinted with permission from Rohatgi, 1999.)

complex. However, purification from 5 calf brains can be handled more easily and should provide enough material for a few hundred assays. Throughout the purification, the Arp2/3 complex is followed by Western blotting with either $\alpha$-Arp3 or $\alpha$-p34ARC IgGs (provided by Dr. Matt Welch of University of California, Berkeley). $\alpha$-Arp2 and $\alpha$-Arp3 antibodies that are commercially available from Santa Cruz Biotech can also be used for this purpose. All chromatography media described here are purchased from Amersham Biosciences. First, freshly harvested calf brains obtained from a local slaughterhouse are stripped of meninges and adherent clots, coarsely chopped, and as much fibrous white matter is removed as possible; it is then quickly washed in ice-cold water. The brain pieces are mixed with 1:1 w/v ice-cold lysis buffer (10 $\mathrm{m} M \mathrm{~K}$-HEPES, pH 7.3, $100 \mathrm{~m} M$ 
$\mathrm{KCl}, 0.4 \mathrm{~m} M$ EDTA, $2 \mathrm{~m} M \mathrm{MgCl}_{2}, 1 \mathrm{~m} M$ PMSF, $10 \mu \mathrm{g} / \mathrm{ml}$ each leupeptin/ pepstatin/chymostatin, and $1 \mathrm{~m} M$ DTT) and blended in a Waring blender (setting 3) for $1 \mathrm{~min}$. The blended material is then fed through a motorized continuous-flow overhead Teflon homogenizer (Yamato). The homogenate is spun at $12,000 \mathrm{~g}$ for $30 \mathrm{~min}$ to remove particulates. The supernatant from this low-speed spin is collected and spun at 100,000 $\mathrm{g}$ for $1 \mathrm{~h}$ to generate the clear, membrane-free bovine brain high-speed supernatant (bovine brain HSS).

Ammonium sulfate is added to $0.8 M$ and the bovine brain HSS is incubated in batch with butyl Sepharose. The flow through, containing most of the Arp $2 / 3$, is precipitated by adding solid ammonium sulfate to bring to a final concentration of $80 \%$ saturation. This precipitation procedure is repeated in several subsequent steps to reduce the volumes of the fractions (see later). The precipitate is collected by centrifugation and dialyzed into DEAE buffer $\left(50 \mathrm{~m} M\right.$ Tris- $\mathrm{HCl}, \mathrm{pH} 7.8,1 \mathrm{~m} M \mathrm{MgCl}_{2}$, $1 \mathrm{~m} M$ DTT, $0.2 \mathrm{~m} M$ ATP) and applied to a DEAE Sepharose column. The flow through contains the Arp $2 / 3$ complex and is precipitated again with ammonium sulfate $(80 \%)$, and the pellet is dialyzed into $\mathrm{S}$ buffer (20 $\mathrm{m} M$ MES, pH6.1, $1 \mathrm{~m} M \mathrm{MgCl}_{2}, 0.2 \mathrm{~m} M$ ATP, $1 \mathrm{~m} M$ DTT). The dialyzed material is loaded onto a SP-Sepharose column and eluted with a linear gradient of $0-350 \mathrm{mM} \mathrm{KCl}$. Fractions containing Arp2/3 are neutralized to $\sim \mathrm{pH} 7.0$ with $1.5 M$ Tris base, pooled, and precipitated with ammonium sulfate $(80 \%)$. The pellet is redissolved in buffer P $(50 \mathrm{mM}$ phosphate, $\mathrm{pH}$ 7.3, $1.2 \mathrm{M}$ ammonium sulfate, $1 \mathrm{~m} M \mathrm{MgCl}_{2}, 0.2 \mathrm{~m} M$ ATP, $1 \mathrm{~m} M$ DTT, $0.5 \mathrm{~m} M$ EDTA), applied to a Resource Phenyl column, and eluted with a linear gradient of 1.2-0 $M$ ammonium sulfate. Again, fractions containing Arp $2 / 3$ complex are precipitated with $80 \%$ ammonium sulfate, resuspended in buffer $\mathrm{F}(20 \mathrm{~m} M$ phosphate, $\mathrm{pH} 7.3,50 \mathrm{~m} M \mathrm{KCl}, 1$ $\mathrm{m} M \mathrm{MgCl}_{2}, 0.2 \mathrm{~m} M$ ATP, $1 \mathrm{~m} M$ DTT), and fractionated on a Superdex 200 gel filtration column. The Arp2/3 complex elutes from the column at $\sim 200 \mathrm{kDa}$ and is $\sim 90 \%$ pure as judged by Gelcode Blue staining (Fig. 4). The protein was supplemented with $10 \%(\mathrm{w} / \mathrm{v})$ sucrose, snap frozen, and stored at $-80^{\circ}$.

\section{Cdc42 and PIP 2 Vesicles}

Preparations of prenylated $\mathrm{Cdc} 42$ - and $\mathrm{PIP}_{2}$-containing vesicles are described in the previous chapter, except that the vesicle stock is made at $10 \mathrm{~m} M$ total lipid. In the purified system (both Reaction \#1 and \#2), we have found that bacterially produced $\mathrm{Cdc} 42$ is also effective at higher concentrations. 
Actin and Pyrene Actin

The purification of actin from rabbit skeletal muscle and the procedure for pyrene labeling have been described by Zigmond (2000).

On the day before the assay, frozen aliquots of actin and pyrene actin (stock concentrations are typically $5-10 \mathrm{mg} / \mathrm{ml}$ ) are thawed, diluted in $\mathrm{G}$ buffer (5 m $M$ Tris- $\mathrm{HCl}, \mathrm{pH} 8.0,0.2 \mathrm{~m} M \mathrm{CaCl}_{2}, 0.2 \mathrm{~m} M$ ATP, $0.2 \mathrm{~m} M$ DTT) to a final protein concentration of $0.5-1 \mathrm{mg} / \mathrm{ml}$ and incubated for $\sim 10 \mathrm{~h}$ (usually done overnight) at $4^{\circ}$ to allow depolymerization. Residual F-actin is removed by centrifugation at $400,000 \mathrm{~g}(100,000 \mathrm{rpm}$ in a Beckman TLA100 rotor) for $1 \mathrm{~h}$. Care must be taken when collecting the supernatant to avoid disrupting the pellet, which appears transparent and glossy. Actin concentrations are determined after centrifugation by the Bradford assay. Our typical recovery is $50-80 \%$.

The final concentration of actin in the polymerization reactions is typically $1-2 \mu M(\sim 35 \%$ pyrene actin). However, because of the variability in the quality of actin and the efficiency of pyrene labeling, the actual amount of actin, as well as the ratio of unlabeled/labeled actin used in the reaction, should be adjusted for each batch of actin/pyrene actin to achieve the optimal signal-to-background ratio.

\section{Sample Reactions}

Reaction \#1: Synergistic activation of recombinant N-WASP with $\mathrm{Cdc} 42$ and $\mathrm{PIP}_{2}$ (Fig. 5)

$39.1 \mu \mathrm{l} \mathrm{H}_{2} \mathrm{O}$

$5.8 \mu \mathrm{l} 10 \times$ Assay buffer $(10 \times: 200 \mathrm{~m} M$ HEPES, pH 7.7, $1 \mathrm{M} \mathrm{KCl}$, $10 \mathrm{~m} M \mathrm{MgCl}_{2}, 1 \mathrm{~m} M$ EDTA, $10 \mathrm{~m} M$ DTT)

$1.2 \mu$ l ATP (10 $\mathrm{m} M$ stock; $0.2 \mathrm{~m} M$ final)

$0.2 \mu$ l Arp2/3 complex (9 $\mu M$ stock; $30 \mathrm{n} M$ final)

$0.6 \mu \mathrm{l}$ recombinant $\mathrm{N}$-WASP (10 $\mu M$ stock; $100 \mathrm{n} M$ final)

$0.5 \mu \mathrm{l} \mathrm{Cdc42-GTP} \gamma \mathrm{S}$ (30 $\mu M$ stock; $250 \mathrm{n} M$ final)

$0.6 \mu \mathrm{l} \mathrm{PIP}_{2}$ vesicles (10 $\mathrm{m} M$ stock; $100 \mu M$ final)

$12.0 \mu \mathrm{l}$ actin/pyrene actin mixture $(10 \mu M$ stock, $65 \%$ unlabeled, $35 \%$ pyrene labeled; $2 \mu M$ final)

60- $\mu$ l reaction

Reaction \#2: Stimulation of native N-WASP-WIP complex with Toca- 1 and $\mathrm{Cdc} 42$ (Fig. 6)

$39.4 \mu 1 \mathrm{H}_{2} \mathrm{O}$

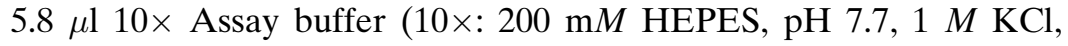
$10 \mathrm{~m} M \mathrm{MgCl}_{2}, 1 \mathrm{~m} M$ EDTA, $10 \mathrm{~m} M$ DTT)

$1.2 \mu \mathrm{l}$ ATP (10 $\mathrm{m} M$ stock; $0.2 \mathrm{~m} M$ final) 


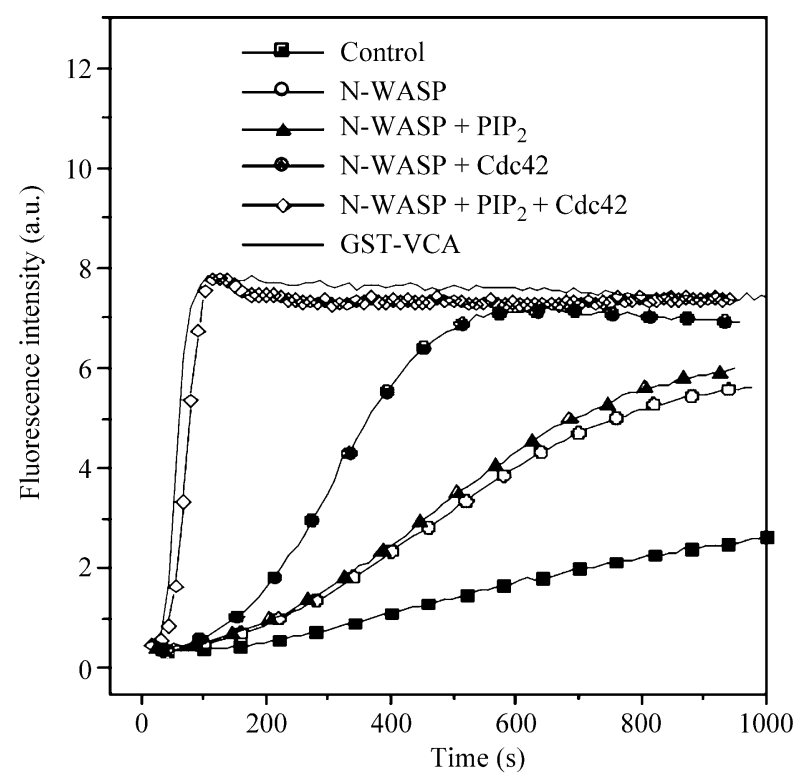

FIG. 5. Synergistic activation of recombinant N-WASP with Cdc42 and $\mathrm{PIP}_{2}$ (Reaction $\# 1)$. The pyrene actin assay used to monitor the effect of Cdc42-GTP $\gamma \mathrm{S}(500 \mathrm{n} M), \mathrm{PI}(4,5) \mathrm{P}_{2^{-}}$ containing vesicles $(100 \mu M, \mathrm{PC}: \mathrm{PI}: \mathrm{PI}(4,5) \mathrm{P} 2,48: 48: 4)$, or both on actin polymerization $(2.5$ $\mu M$ total actin, $40 \%$ pyrene labeled, and $60 \mathrm{n} M$ Arp $2 / 3$ complex) stimulated by N-WASP (200 $\mathrm{n} M)$. The solid line shows stimulation of actin polymerization under the same conditions by a GST fusion of VCA $(200 \mathrm{n} M)$, a C-terminal fragment of N-WASP that is constitutively active in stimulating the Arp2/3 complex. Note that the concentrations of reaction components shown in this figure are slightly different from the sample reaction given in the text, but the general properties of the reactions remain similar. (Reprinted with permission from Rohatgi, 1999.)

$0.2 \mu \mathrm{l}$ Arp2/3 complex (9 $\mu M$ stock; $30 \mathrm{n} M$ final)

$0.6 \mu \mathrm{l}$ native N-WASP-WIP complex (0.6 $\mu M$ stock; $6 \mathrm{n} M$ final)

$0.3 \mu \mathrm{l}$ Toca-1 (2 $\mu M$ stock; $10 \mathrm{n} M$ final)

$0.5 \mu \mathrm{l} \mathrm{Cdc42-GTP} \gamma \mathrm{S}$ (30 $\mu M$ stock; $250 \mathrm{n} M$ final)

$12.0 \mu \mathrm{l}$ actin/pyrene actin mixture (10 $\mu M$ stock, $65 \%$ unlabeled, $35 \%$ pyrene labeled; $2 \mu M$ final)

\section{0- $\mu$ l reaction}

Protein stocks are typically prepared in $1 \times$ assay buffer $(20 \mathrm{mM}$ HEPES, pH 7.7, $100 \mathrm{~m} M \mathrm{KCl}, 1 \mathrm{~m} M \mathrm{MgCl}_{2}, 0.1 \mathrm{~m} M$ EDTA, $1 \mathrm{~m} M$ DTT) or in other buffers with similar compositions by dialysis or dilution. The volumes of the reactions components, as well as the amount of $10 \times$ assay buffer needed per reaction to make the final buffer concentration $1 \times$ 
A

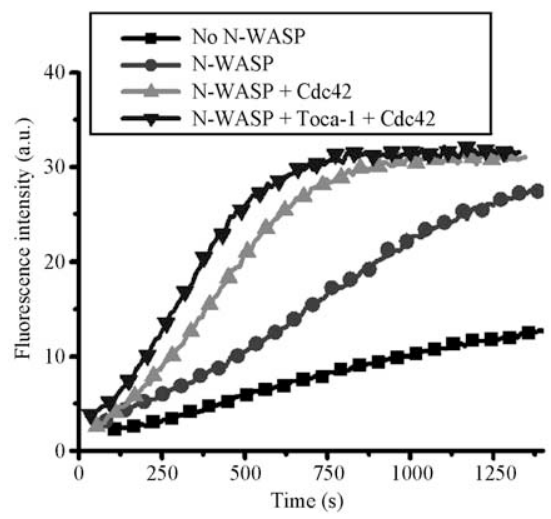

B

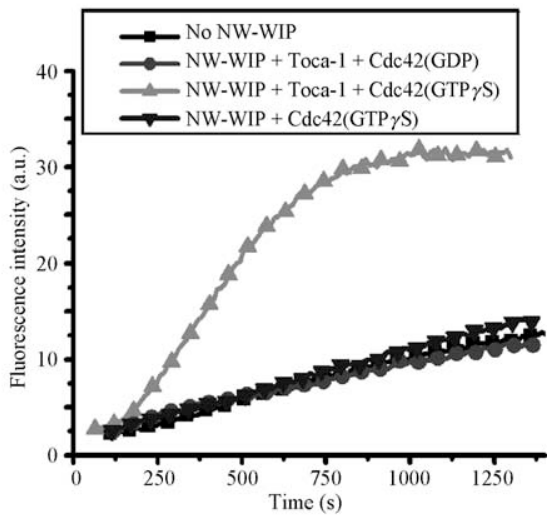

FIG. 6. Stimulation of native N-WASP-WIP complex with Toca-1 and Cdc42 (Reaction \#2). (A) The pyrene actin assay used to determine the effect of Toca-1 $(10 \mathrm{n} M)$ on actin polymerization ( $2 \mu M$ total G-actin; $35 \%$ pyrene labeled) in the presence Arp $2 / 3$ complex (30 $\mathrm{n} M)$, recombinant N-WASP $(100 \mathrm{n} M)$, and Cdc42-GTP $\gamma \mathrm{S}(250 \mathrm{n} M)$. (B) The pyene actin assay used to compare the activation of the N-WASP-WIP complex (NW-WIP, $6 \mathrm{n} M$ ) by Cdc42-GTP $\gamma \mathrm{S}(250 \mathrm{n} M)$ in the presence or absence of Toca-1 $(10 \mathrm{n} M)$. Cdc42-GDP is completely inactive in stimulating actin polymerization under the same conditions. Note the different behaviors of recombinant N-WASP and native N-WASP-WIP in response to Cdc42 and Toca-1 stimulation. (Reprinted with permission from Ho et al., 2004.)

assay buffer, need to be adjusted according to the actual concentrations the protein stocks. All reaction components except actin/pyrene actin are first mixed together in the order listed above and incubated for $5 \mathrm{~min}$ at room temperature. The reaction is initiated by adding a mixture of actin and pyrene actin to the preincubated reaction, which is then immediately transferred into a quartz cuvette (Varian Sub-Micro Fluorometer Cell, $40 \mu \mathrm{l}$ ) and monitored in a fluorescence spectrophotometer (Varian Cary Eclipse) using the provided kinetics software with the temperature control set to $20^{\circ}$. Pyrene fluorescence is measured at $407 \mathrm{~nm}$ with excitation at $365 \mathrm{~nm}$. With the Varian Cary Eclipse equipped with a Peltier Multicell Holder, four independent actin polymerization reactions can be monitored simultaneously.

In contrast to the extract system, which possesses a high actin-buffering capacity because of the presence of a large number of actin-binding proteins, the purified system lacks such capacity and is prone to a variety of experimental perturbations. For example, the baseline level of actin polymerization in the absence of any stimulatory factor can vary significantly, depending the preparation of the actin, and even within the same actin 
preparation, it can vary from day to day. On the basis of our experience, at least part of this variability seems to originate from the procedures of the overnight depolymerization and the subsequent removal of residual actin by centrifugation. Given these considerations, proper controls, including the actin alone baseline reaction, must be conducted at the beginning of each day of the assay, and curve-to-curve comparisons should only be made with results obtained using the same actin stock within the same day. Other important controls to account for baseline levels of N-WASP and Arp2/3 activity include the actin + Arp2/3 reaction, the actin + Arp2/3 + N-WASP (or N-WASP-WIP complex) reaction, and the actin + Arp $2 / 3+$ agonists (Cdc42) without N-WASP reaction (Figs. 5 and 6).

Kinetic analyses of actin polymerization reactions can be performed using the software provided by the fluorescence spectrophotometer or by using other graphing programs such as Origin (MicroCal Software) or Excel (Microsoft). Polymerization curves can be quantified by the following methods: (1) measuring the slope of the linear, elongating phase of the actin assembly curve; (2) calculating the number of barbed ends from the rate of elongation; and (3) calculating the half-time to steady state. A detailed discussion of these analytical methods can be found in an earlier volume of Methods in Enzymology (Mullins and Machesky, 2000).

\section{Properties of the Purified System}

The ultimate success and utility of a purified and reconstituted signaling system is its ability to recapitulate the behavior of the pathway in complex extracts (or better yet, cells). Although the components connecting Cdc42 to actin nucleation through the Arp2/3 complex described in Reaction \#1 and \#2 have not been systematically analyzed in vivo, we believe that Reaction \#2 represents a more complete reconstitution of the pathway in the extracts. Reaction \#1 captures a cardinal feature of pure N-WASP, its ability to be synergistically activated by two upstream activators, Cdc42 and PIP $_{2}$. However, there are critical differences between this system and the extract system. Most importantly, nearly all the N-WASP in extracts is present as a complex with WIP/CR16, and unlike recombinant N-WASP, this complex exhibits zero basal activity in the absence of upstream stimuli (as one would expect for a central regulator of actin polymerization). Reaction \#2 addresses many of these inconsistencies by showing that the purified native N-WASP-WIP complex has no detectable level of basal activation and is also completely insensitive to stimulation by $\mathrm{Cdc} 42$ alone. However, it can be activated in a switchlike fashion by Cdc42 and Toca-1, a novel factor that we recently purified from extracts as an essential component of the pathway (Ho et al., 2004). 
A number of issues regarding the $\mathrm{Cdc} 42$-actin signaling pathway remain to be explained. For example, the precise biochemical mechanism by which the N-WASP-WIP complex is activated by Toca- 1 and Cdc42, as well the regulation of Toca- 1 itself by other signals remain open questions. We have demonstrated that Toca- 1 is a limiting component in extracts, and we expect the levels/activity of Toca-1 to be an important regulator of flux through this pathway (Ho et al., 2004). Finally, systematic testing of these components in in vivo systems will be crucial for understanding the cellular function of the Cdc42 pathway. It is our hope that the extract and purified assay systems described in these two chapters will continue to serve as powerful tools for studies of signal-dependent actin regulation, because they provide a way to biochemically dissect signal transduction pathways at a resolution that is difficult to achieve in cell-based assays.

\section{Acknowledgment}

We thank Dr. Le Ma for his comments on the manuscript. The studies described in this chapter were supported by grants from National Institutes of Health to M. W. K.

\section{References}

Carlier, M. F., Nioche, P., Broutin-L'Hermite, I., Boujemaa, R., Le Clainche, C., Egile, C., Garbay, C., Ducruix, A., Sansonetti, P., and Pantaloni, D. (2000). GRB2 links signaling to actin assembly by enhancing interaction of neural Wiskott-Aldrich syndrome protein (N-WASp) with actin-related protein (ARP2/3) complex. J. Biol. Chem. 275, 21946-21952.

Cooper, J. A., Walker, S. B., and Pollard, T. D. (1983). Pyrene actin: Documentation of the validity of a sensitive assay for actin polymerization. J. Muscle Res. Cell Motil. 4, 253-262.

Cory, G. O., Garg, R., Cramer, R., and Ridley, A. J. (2002). Phosphorylation of tyrosine 291 enhances the ability of WASp to stimulate actin polymerization and filopodium formation. Wiskott-Aldrich Syndrome protein. J. Biol. Chem. 277, 45115-45121.

Egile, C., Loisel, T. P., Laurent, V., Li, R., Pantaloni, D., Sansonetti, P. J., and Carlier, M. F. (1999). Activation of the CDC42 effector N-WASP by the Shigella flexneri IcsA protein promotes actin nucleation by Arp2/3 complex and bacterial actin-based motility. J. Cell Biol. 146, 1319-1332.

Higgs, H. N., and Pollard, T. D. (2000). Activation by Cdc42 and PIP(2) of Wiskott-Aldrich syndrome protein (WASp) stimulates actin nucleation by Arp2/3 complex. J. Cell Biol. 150, 1311-1320.

Ho, H. Y., Rohatgi, R., Lebensohn, A. M., Ma, L., Li, J., Gygi, S. P., and Kirschner, M. W. (2004). Toca-1 mediates Cdc42-dependent actin nucleation by activating the N-WASPWIP complex. Cell 118, 203-216.

Ho, H. Y., Rohatgi, R., Ma, L., and Kirschner, M. W. (2001). CR16 forms a complex with NWASP in brain and is a novel member of a conserved proline-rich actin-binding protein family. Proc. Natl. Acad. Sci. USA 98, 11306-11311.

Kouyama, T., and Mihashi, K. (1980). Pulse-fluorometry study on actin and heavy meromyosin using F-actin labelled with N-(1-pyrene)maleimide. Eur. J. Biochem. 105, 279-287. 
Ma, L., Rohatgi, R., and Kirschner, M. W. (1998). The Arp2/3 complex mediates actin polymerization induced by the small GTP-binding protein Cdc42. Proc. Natl. Acad. Sci. USA 95, 15362-15367.

Machesky, L. M., Atkinson, S. J., Ampe, C., Vandekerckhove, J., and Pollard, T. D. (1994). Purification of a cortical complex containing two unconventional actins from Acanthamoeba by affinity chromatography on profilin-agarose. J. Cell Biol. 127, 107-115.

Miki, H., Miura, K., and Takenawa, T. (1996). N-WASP, a novel actin-depolymerizing protein, regulates the cortical cytoskeletal rearrangement in a PIP2-dependent manner downstream of tyrosine kinases. EMBO J. 15, 5326-5335.

Mullins, R. D., and Machesky, L. M. (2000). Actin assembly mediated by Arp2/3 complex and WASP family proteins. Methods Enzymol. 325, 214-237.

Ramesh, N., Anton, I. M., Hartwig, J. H., and Geha, R. S. (1997). WIP, a protein associated with Wiskott-Aldrich syndrome protein, induces actin polymerization and redistribution in lymphoid cells. Proc. Natl. Acad. Sci. USA 94, 14671-14676.

Rohatgi, R., Ho, H. Y., and Kirschner, M. W. (2000). Mechanism of N-WASP activation by CDC42 and phosphatidylinositol 4, 5-bisphosphate. J. Cell Biol. 150, 1299-1310.

Rohatgi, R., Ma, L., Miki, H., Lopez, M., Kirchhausen, T., Takenawa, T., and Kirschner, M. W. (1999). The interaction between N-WASP and the Arp2/3 complex links Cdc42dependent signals to actin assembly. Cell 97, 221-231.

Rohatgi, R., Nollau, P., Ho, H. Y., Kirschner, M. W., and Mayer, B. J. (2001). Nck and phosphatidylinositol 4,5-bisphosphate synergistically activate actin polymerization through the N-WASP-Arp2/3 pathway. J. Biol. Chem. 276, 26448-26452.

Suetsugu, S., Hattori, M., Miki, H., Tezuka, T., Yamamoto, T., Mikoshiba, K., and Takenawa, T. (2002). Sustained activation of N-WASP through phosphorylation is essential for neurite extension. Dev. Cell 3, 645-658.

Torres, E., and Rosen, M. K. (2003). Contingent phosphorylation/dephosphorylation provides a mechanism of molecular memory in WASP. Mol. Cell 11, 1215-1227.

Welch, M. D., and Mitchison, T. J. (1998). Purification and assay of the platelet Arp2/3 complex. Methods Enzymol. 298, 52-61.

Zigmond, S. H. (2000). In vitro actin polymerization using polymorphonuclear leukocyte extracts. Methods Enzymol. 325, 237-254.

\title{
[15] Biochemical Analysis of Mammalian Formin Effects on Actin Dynamics
}

\author{
By Elizabeth S. Harris and Henry N. Higgs
}

\begin{abstract}
Formins are members of a conserved family of proteins, present in all eukaryotes, that regulate actin dynamics. Mammals have 15 distinct formin genes. From studies to date, surprising variability between these isoforms has been uncovered. All formins examined have several common effects on actin dynamics in that they: (1) accelerate nucleation rate; (2) alter filament barbed end elongation/depolymerization rates; and (3) antagonize capping
\end{abstract}

